\title{
Note on the Distribution of Ramanujan's Tau Function
}

\author{
By D. H. Lehmer
}

Abstract. According to a conjecture of Sato and Tate, the angle $\theta$ whose cosine is $\frac{1}{2} \tau(p) p^{-11 / 2}$, where $\tau$ is Ramanujan's function and $p$ a prime, is distributed over $[0, \pi]$ according to a $\sin ^{2} \theta$ law. The paper reports on a test of this conjecture for the 1229 primes under 10000. Extreme values of $\theta$ are also given.

Ramanujan's function $\tau(n)$ is defined by its generator

$$
\sum_{n=1}^{\infty} \tau(n) x^{n}=x\left\{(1-x)\left(1-x^{2}\right)\left(1-x^{3}\right) \cdots\right\}^{24} \text {. }
$$

For $p$ a prime we can define $\theta_{p}$ by

$$
\tau(p)=2 p^{11 / 2} \cos \theta_{p}
$$

According to the famous Ramanujan conjecture, $\theta_{p}$ should be real. Assuming this, one can ask how are the values of $\theta_{p}$ distributed in the interval $0<\theta<\pi$. Sato and Tate [1] have conjectured that the density of those primes $p$ for which

is given by

$$
a<\theta_{p}<b
$$

$$
\frac{2}{\pi} \int_{a}^{b} \sin ^{2} \theta d \theta .
$$

In other words, if we define $I(t)$ by

$$
I(t)=\frac{2}{\pi} \int_{\operatorname{arcos} t}^{\pi / 2} \sin ^{2} \theta d \theta
$$

then the density of primes $p$ for which

$$
A<\frac{1}{2} \tau(p) p^{-11 / 2}<B
$$

is $I(B)-I(A)$.

To test this conjecture we computed $\frac{1}{2} \tau(p) p^{-11 / 2}$ for each of the 1229 primes $p<10^{4}$ from our table of $\tau(n)$ [2] and made a histogram of their values. If we divide the interval $(-1,1)$ into equal subintervals of length $h$ we can count the number $N_{k}(h)$ of primes $p$ for which $\cos \theta_{p}$ satisfies

$$
k h \leqq \cos \theta_{p}<(k+1) h .
$$

According to the conjecture $N(h)$ should be approximately the expected number:

$$
E_{k}(h)=1229\{I((k+1) h)-I(k h)\} .
$$

Received December 1, 1969.

AMS Subject Classifications. Primary 1020, 1063.

Key Words and Phrases. Ramanujan's tau function, distribution.

Copyright @ 1971, American Mathematical Society 
Thus, for $h=1$ we are merely asking how many $\tau(p)$ are positive and how many are negative. The expected number in each case is just one half of the total number of primes, namely 614.5 . The actual counts give 616 positive and 613 negative values.

Breaking $(-1,1)$ into four parts we have $\left(h=\frac{1}{2}\right)$

\begin{tabular}{rrr}
\multicolumn{1}{c}{$K$} & $N_{K}\left(\frac{1}{2}\right)$ & $E_{K}\left(\frac{1}{2}\right)$ \\
\hline-2 & 228 & 240 \\
-1 & 385 & 374 \\
0 & 374 & 374 \\
1 & 242 & 240
\end{tabular}

For $h=\frac{1}{4}$ we find

\begin{tabular}{rrr}
\multicolumn{1}{c}{$K$} & $N_{K}\left(\frac{1}{4}\right)$ & $E_{K}\left(\frac{1}{4}\right)$ \\
\hline-4 & 89 & 89 \\
-3 & 139 & 152 \\
-2 & 187 & 181 \\
-1 & 198 & 194 \\
0 & 189 & 194 \\
1 & 185 & 181 \\
2 & 155 & 152 \\
3 & 87 & 89
\end{tabular}

For $h=\frac{1}{8}$ we find

\begin{tabular}{|c|c|c|c|c|c|}
\hline$K$ & $N_{K}\left(\frac{1}{8}\right)$ & $E_{K}\left(\frac{1}{8}\right)$ & $K$ & $N_{K}\left(\frac{1}{8}\right)$ & $E_{K}\left(\frac{1}{8}\right)$ \\
\hline-8 & 29 & 32 & 0 & 91 & 98 \\
\hline-7 & 60 & 57 & 1 & 98 & 96 \\
\hline-6 & 69 & 71 & 2 & 99 & 93 \\
\hline-5 & 70 & 81 & 3 & 86 & 88 \\
\hline-4 & 89 & 88 & 4 & 81 & 81 \\
\hline-3 & 98 & 93 & 5 & 74 & 71 \\
\hline-2 & 87 & 96 & 6 & 63 & 57 \\
\hline-1 & 111 & 98 & 7 & 24 & 32 \\
\hline
\end{tabular}

The agreement here is pretty reassuring.

With reference to the original Ramanujan conjecture we submit a list of those primes $p$ whose $\cos \theta_{\nu}$ is larger (or smaller) than those of all smaller primes. These extreme primes and their values are as follows. 
Extremely large

\begin{tabular}{r|c}
$p$ & $\left|\cos \theta_{p}\right|$ \\
\hline 2 & .26516 \\
3 & .29936 \\
5 & .34560 \\
11 & .50043 \\
17 & .58982 \\
47 & .85458 \\
103 & .95940 \\
3371 & .97489 \\
3967 & .97571 \\
7451 & .97751 \\
7589 & .98026
\end{tabular}

Extremely small

\begin{tabular}{r|c}
\multicolumn{1}{c|}{$p$} & $\left|\cos \theta_{p}\right|$ \\
\hline 2 & .26516 \\
7 & .18827 \\
31 & .16575 \\
43 & .00888 \\
617 & .00547 \\
907 & .00228 \\
2927 & .00049 \\
7993 & .00031
\end{tabular}

Department of Mathematics

\section{University of California}

Berkeley, California 94720

1. Letter of J. P. Serre to author, June 1, 1964.

2. D. H. LEHMER, Table of Ramanujan's Function $\tau(n)$, 1963. Ms. of 164 pages of computer printout. UMT File. 